REVISTA CIENCIAS BIOMÉDICAS

GUÍA DE MANEJO Y PROTOCOLOS

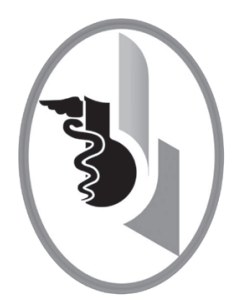

\title{
VIGILANCIA FETAL DURANTE EL TRABAJO DE PARTO
}

\author{
FETAL MONITORING DURING THE OBSTETRIC LABOR
}

\author{
Salcedo-Ramos Francisco ${ }^{1}$ \\ Méndez-Rodríguez Rogelio ${ }^{1}$ \\ Vallejo-Navarro Carolina ${ }^{2}$
}

Correspondencia: fjsalcedo@yahoo.com

Recibido para evaluación: noviembre-21-2014. Aceptado para publicación: mayo-26-2015.

\section{RESUMEN}

La monitoria fetal intraparto hace parte de las pruebas para la valoración del bienestar fetal. Es método de fácil realización e interpretación, no invasivo, de bajo costo, reproducible, sin efectos secundarios ni contraindicaciones. El objetivo fundamental del estudio es la evaluación del estado fetal durante el parto, identificando los fetos que posiblemente puedan estar comprometidos. Ello se determina por alteraciones en las siguientes variables. [A] Frecuencia, variabilidad y desaceleraciones del ritmo cardíaco fetal. [B] Movimientos fetales. [C] Cambios en la actividad cardíaca en relación con la actividad uterina. Los hallazgos permiten clasificar el estado fetal en tres categorías. Así se pueden establecer medidas oportunas antes que se produzcan daños irreversibles en el feto o en el peor de los casos la muerte fetal. Rev.cienc.biomed. 2015;6(1):170178

PALABRAS CLAVE

Cardiotocografia; monitoreo fetal; Frecuencia cardiaca fetal; Trabajo de parto.

\section{SUMMARY}

The intrapartum fetal monitoring is part of the tests to assess the fetal well-being. It is an easy, cheap, reproducible and non-invasive method without adverse effects or contraindications. The main objective of this method is to assess the fetal status during the obstetric labor, identifying the fetus that probably could have alterations. It is determined for alterations in the next variables: [A] Frequency, variability and decelerations of the fetal cardiac rhythm [B] Fetal movements. [C] Changes in the cardiac activity according to the uterine activity. The findings allow classifying the fetal status in three categories. And in this way, the adequate measures could be established before irreversible lesions and death could occur in the fetus. Rev.cienc.biomed. 2015;6(1):170-178

\section{KEYWORDS}

Cardiotocography; Fetal Monitoring; Heart fetal rate; Obstetric labor.

\footnotetext{
1 Médico. Especialista en Ginecología y Obstetricia. Docente Departamento de Ginecología y Obstetricia. Facultad de Medicina. Universidad de Cartagena. Colombia.

2 Médico. Estudiante de Postgrado. Ginecología y Obstetricia. Facultad de Medicina. Universidad de Cartagena. Colombia.
} 


\section{INTRODUCCIÓN}

La vigilancia fetal durante el trabajo de parto es uno de los métodos recomendados en la asistencia durante la fase activa del período de dilatación y borramiento. En la actualidad para el control de la frecuencia cardíaca fetal (FCF) la mayoría de los instrumentos de auscultación son externos. Los más comunes incluyen la monitorización electrónica cardiotocográfica continua o intermitente y el uso de la auscultación con fonendoscopio en los embarazos de bajo riesgo. La Guia de Atención Integral del Ministerio de Protección Social de Colombia, recomienda sus usos como métodos válidos de vigilancia fetal durante el trabajo de parto.

El fin de vigilar la actividad cardíaca fetal, es identificar precozmente alteraciones del bienestar fetal como producto de oxigenación inadecuada y reconocer tempranamente el estado fetal insatisfactorio o no tranquilizador, nuevo término que reemplaza a su- frimiento fetal. Ello permite tomar decisiones clínicas en beneficio del feto, obteniendo recién nacidos en mejores condiciones clínicas y reducción en la morbimortalidad neonatal.

El objetivo es difundir la clasificación de la monitorización fetal electrónica intraparto publicada por el Colegio Americano de Obstetras y Ginecólogos y recomendar de acuerdo a las evidencias actuales, las acciones a seguir ante cada uno de los resultados (Macones GA, Hankins G, Spong C. 2008).

\section{MONITOREO FETAL INTERMITENTE IN- TRAPARTO}

La monitorización fetal intraparto intermitente con fonendoscopio o equipo doppler se considera segura en pacientes de bajo riesgo. No incrementa las secuelas neurológicas y disminuye las cesáreas hasta en un $40 \%$ si se compara con el método electrónico continuo (Tabla $\mathrm{N}^{\circ} 1$ ).

\begin{tabular}{|c|c|c|}
\hline \multicolumn{3}{|c|}{$\begin{array}{c}\text { TABLA } N^{\circ} 1 . \\
\text { MONITOREO FETAL INTERMITENTE INTRAPARTO }\end{array}$} \\
\hline ¿CUÁNDO SE REALIZA? & ¿CÓMO SE INTERPRETA? & ¿CÚAL ES LA CONDUCTA? \\
\hline $\begin{array}{l}\text { - En el primer periodo del } \\
\text { trabajo de parto. } \\
\text { - [A] En fase latente, cada } 30 \\
\text { minutos durante un minuto } \\
\text { y después de la contracción } \\
\text { uterina. } \\
\text { - [B] En fase activa, cada } 15 \\
\text { minutos durante un minuto } \\
\text { y después de contracción. } \\
\text { uterina. }\end{array}$ & $\begin{array}{l}\text { - Normal } \\
\text { [A] FCF entre } 110 \text { y } 160 \\
\text { latidos por minuto. }\end{array}$ & \multirow{2}{*}{$\begin{array}{l}\text { - Interpretación anormal } \\
\text { [A] Coloque a la paciente en } \\
\text { decúbito lateral izquierdo. } \\
\text { [B] Evalúe los signos vitales } \\
\text { maternos. Frecuencia cardíaca, } \\
\text { temperatura y tensión arterial. } \\
\text { [C] Si hay hipotensión arterial inicie } \\
\text { un bolo de } 500 \text { cc de cristaloides. } \\
\text { [D] Si hay fiebre administrar un } \\
\text { gramo de acetaminofén vía oral. } \\
\text { [E] Realizar tacto vaginal para } \\
\text { constatar la dilatación y descartar } \\
\text { prolapso de cordón umbilical. } \\
\text { [F] Realizar monitoreo electrónico } \\
\text { intraparto para evaluar la } \\
\text { persistencia de las alteraciones } \\
\text { de la frecuencia cardíaca fetal las } \\
\text { desaceleraciones. }\end{array}$} \\
\hline $\begin{array}{l}\text { - En el segundo periodo del } \\
\text { trabajo de parto o expulsivo. } \\
\text { [A] Cada } 5 \text { minutos durante } \\
\text { un minuto y luego de la } \\
\text { contracción uterina. }\end{array}$ & $\begin{array}{l}\text { - Anormal } \\
\text { - }[\mathrm{A}] \text { Taquicardia fetal. FCF } \\
\text { superior a } 160 \text { latidos por } \\
\text { minuto. } \\
\text { - } \text { [B] Bradicardia fetal. } \\
\text { - FCF inferior a } 110 \text { latidos } \\
\text { por minuto o identificación } \\
\text { de desaceleraciones en el } \\
\text { ritmo cardíaco fetal. }\end{array}$ & \\
\hline
\end{tabular}

\section{MONITOREO FETAL CONTINUO INTRAPARTO}

El monitoreo fetal continuo se debe adelantar con una técnica correcta para evitar falsos positivos o negativos. La posición de la paciente debe ser semisentada o decúbito lateral, siempre evitar el decúbito supino, ya que se comprime la vena cava y la arteria aorta, afectándose la perfusión uteroplacentaria. La paciente no debe estar en ayunas ya que ello afecta la variabilidad en la frecuencia cardíaca y los movimientos fetales. La duración del estudio debe ser mínimo de 
20 minutos, evitando la administración simultánea de medicamentos con efecto sedante. El monitoreo fetal se recomienda en embarazos con 32 o más semanas de gestación.

La monitorización de la actividad cardíaca fetal se realizará siempre externamente, la única excepción para adelantar monitorización interna, son los casos de alto riesgo en la que la calidad del registro sea insuficiente para su evaluación.

Las siguientes son indicaciones para monitorización fetal continua. [A] Embarazadas de alto riesgo. [B] Presencia de sangrado vaginal durante el trabajo de parto. [C] Infección intrauterina o corioamnionitis. [D] Inducción y conducción del trabajo de parto. [E] Hipertonía uterina. [F] Embarazo de 41 semanas. [G] Líquido amniótico meconiado. [H] Embarazadas de bajo riesgo que presenten alteraciones de la fetocardia. El monitoreo fetal continuo durante el trabajo de parto, puede pasar a ser realizado cada hora, cuando existen dificultades institucionales y los resultados encontrados han sido normales.

Las variables que se analizan en el monitoreo fetal continuo intraparto son las siguientes: [A] Contracciones uterinas. [B] FCF basal. [C] Variabilidad de la FCF basal. [D] Presencia de aceleraciones. [C] Presencia de desaceleraciones periódicas o episódicas (Tabla $\mathrm{N}^{\circ} 2$ ).

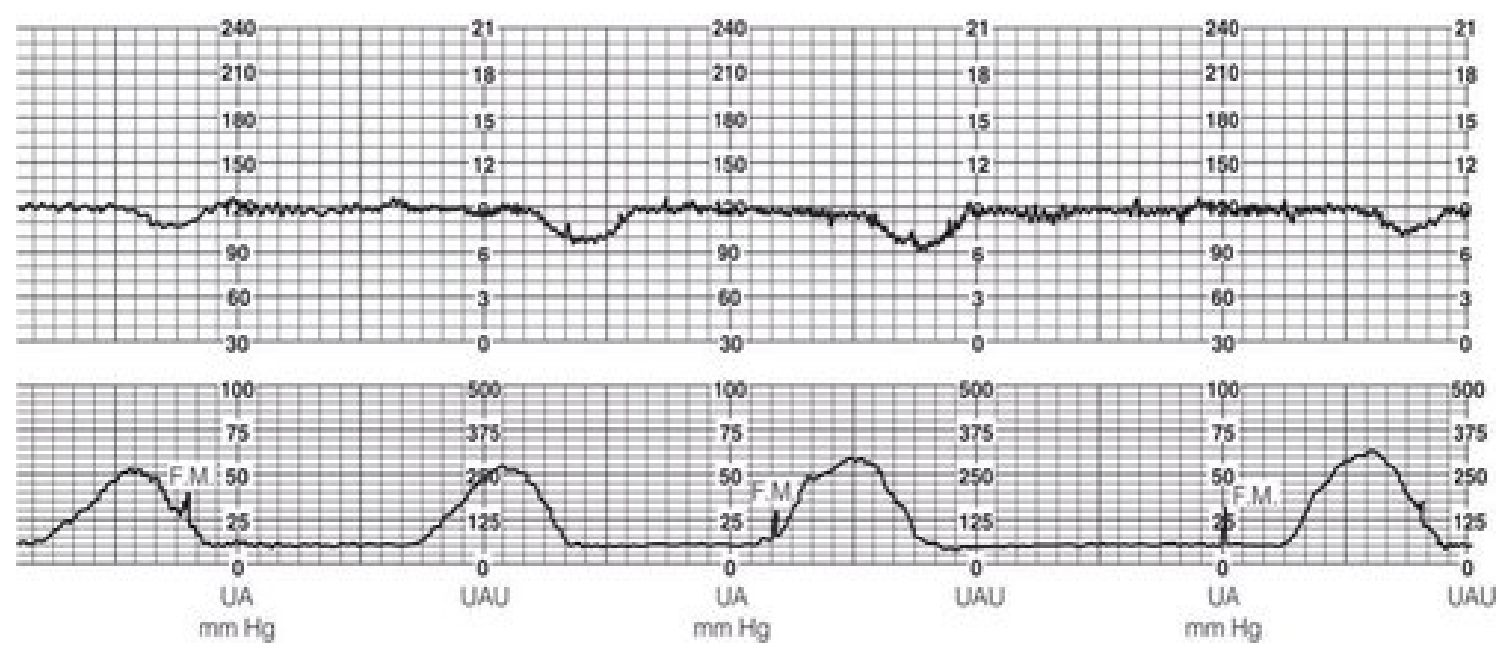

Figura $\mathbf{N}^{\circ}$ 1. Ausencia de la variabilidad de la frecuencia cardíaca fetal

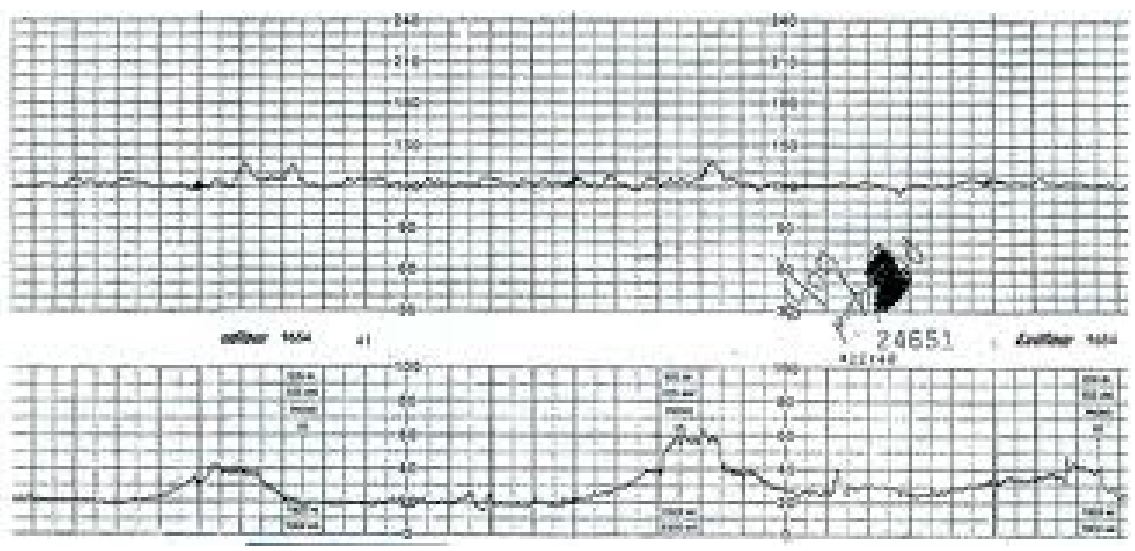

Figura $\mathbf{N}^{\circ}$ 2. Disminución de la variabilidad de la frecuencia cardíaca fetal 


\begin{tabular}{|c|c|c|c|c|}
\hline \multicolumn{5}{|c|}{$\begin{array}{c}\text { TABLA N' } 2 . \\
\text { MONITOREO FETAL CONTINUO INTRAPARTO }\end{array}$} \\
\hline $\begin{array}{l}\text { CONTRACCIONES } \\
\text { UTERINAS }\end{array}$ & FCF BASAL & $\begin{array}{l}\text { VARIABILIDAD DE } \\
\text { LA FCF BASAL }\end{array}$ & $\begin{array}{l}\text { ACELERACIONES DE } \\
\text { LA FCF BASAL }\end{array}$ & $\begin{array}{l}\text { DESACELERACIONES } \\
\text { DE LA FCF BASAL }\end{array}$ \\
\hline $\begin{array}{l}\text { Contar el número } \\
\text { de contracciones } \\
\text { cada diez } \\
\text { minutos. }\end{array}$ & $\begin{array}{l}\text { Observar FCF en } \\
\text { diez minutos. }\end{array}$ & $\begin{array}{l}\text { Son las } \\
\text { fluctuaciones de } \\
\text { la FCF basal. Se } \\
\text { observan como } \\
\text { irregularidades } \\
\text { en la amplitud y } \\
\text { frecuencia de los } \\
\text { latidos cardíacos. }\end{array}$ & \multirow{3}{*}{$\begin{array}{l}\text { Es la elevación } \\
\text { de la FCF en } 15 \\
\text { latidos durante } 15 \\
\text { segundos pero con } \\
\text { duración menor de } \\
2 \text { minutos ( a partir } \\
\text { de la semana } 32 \text { ) } \\
\text { Aceleración antes } \\
\text { de las } 32 \text { semanas } \\
\text { es aumento de } 10 \\
\text { latidos durante } 10 \\
\text { segundos durante } \\
\text { un tiempo menor a } \\
2 \text { minutos. }\end{array}$} & \multirow{3}{*}{$\begin{array}{l}\text { [A] DIPS I } \\
\text { Desaceleración } \\
\text { temprana. Descenso } \\
\text { gradual con } \\
\text { recuperación de la FCF, } \\
\text { el cual es simétrico con } \\
\text { la contracción uterina. } \\
\text { No se asocia a hipoxia } \\
\text { fetal } \\
\text { (Figura No4). } \\
\text { [B] DIPS II } \\
\text { Desaceleración tardía. } \\
\text { Caída gradual de la FCF } \\
\text { en donde el pico de la } \\
\text { contracción uterina no } \\
\text { coincide con el nadir de } \\
\text { la FCF. Se presenta } 30 \\
\text { segundos después. Se } \\
\text { asocia a insuficiencia } \\
\text { uteroplacentaria o } \\
\text { hipoxia fetal. } \\
\text { (Figura No } 5 \text { ). } \\
\text { [C] DIPS-III } \\
\text { Desaceleración } \\
\text { variable. Caída de } \\
\text { la FCF 15 latidos } \\
\text { o más durante } 15 \\
\text { segundos pero menos } \\
\text { de dos minutos. } \\
\text { Puede o no asociarse } \\
\text { a las contracciones } \\
\text { uterinas. Indica posible } \\
\text { compresión del cordón } \\
\text { umbilical. } \\
\text { (Figura No 6). } \\
\text { [D] Desaceleraciones } \\
\text { prolongadas. Caída } \\
\text { de la FCF igual o } \\
\text { mayor a 15 latidos, } \\
\text { con duración entre } \\
\text { 2-10 minutos. Si dura } \\
\text { más de } 10 \text { minutos, } \\
\text { es cambio de línea de } \\
\text { base. } \\
\text { [E] Patrón sinusoidal. } \\
\text { Onda sinusoidal de } \\
\text { la FCF, frecuencia } \\
\text { de } 3-5 \text { ciclos por } \\
\text { minuto. Persiste por } 20 \\
\text { minutos o más. }\end{array}$} \\
\hline \multirow[b]{2}{*}{$\begin{array}{l}\text { [A] Normal: entre } \\
2 \text { y } 5 \\
\text { [B] Taquisistolia: } \\
\text { más de } 5 \\
\text { [C] Bradisistolia: } \\
\text { menos de } 2 \\
\text { [D] Hipertonía: } \\
\text { aumento del } \\
\text { tono uterino } \\
\text { sobre } 12 \mathrm{~mm} / \\
\mathrm{Hg} \text { y no regresa } \\
\text { a la basal entre } \\
\text { contracciones. }\end{array}$} & & $\begin{array}{l}\text { Normal: 6- } 25 \\
\text { latidos por } \\
\text { minuto. } \\
\text { Se puede alterar: } \\
\text { [A] Sueño fetal } \\
\text { [B] Fármacos: } \\
\text { sulfato de } \\
\text { magnesio, } \\
\text { betametasona, } \\
\text { narcóticos, } \\
\text { sedantes, beta } \\
\text { miméticos } \\
\text { [C] Pretérmino } \\
\text { [D] Fetos con } \\
\text { malformaciones } \\
\text { congénitas. } \\
\end{array}$ & & \\
\hline & $\begin{array}{l}\text { Normal de } 110 \\
\text { a } 160 \text { Se puede } \\
\text { incrementar: } \\
\text { [A] Fiebre } \\
\text { [B] Fármacos: } \\
\text { terbutalina, } \\
\text { nifedipino } \\
\text { [C] Fetos con } \\
\text { malforaciones } \\
\text { [D] Pretérmino. }\end{array}$ & $\begin{array}{l}\text { Clasificación } \\
\text { según latidos por } \\
\text { minuto: } \\
\text { [A] Ausente: 0-2 } \\
\text { (Figuras No1), } \\
\text { [B] Mínima: } 3-5 \\
\text { (Figura N²) } \\
\text { [C] Moderada: } \\
6-25 \\
\text { (Figura No 3) } \\
\text { [D] Marcada: } 26 \\
\text { y más }\end{array}$ & & \\
\hline
\end{tabular}



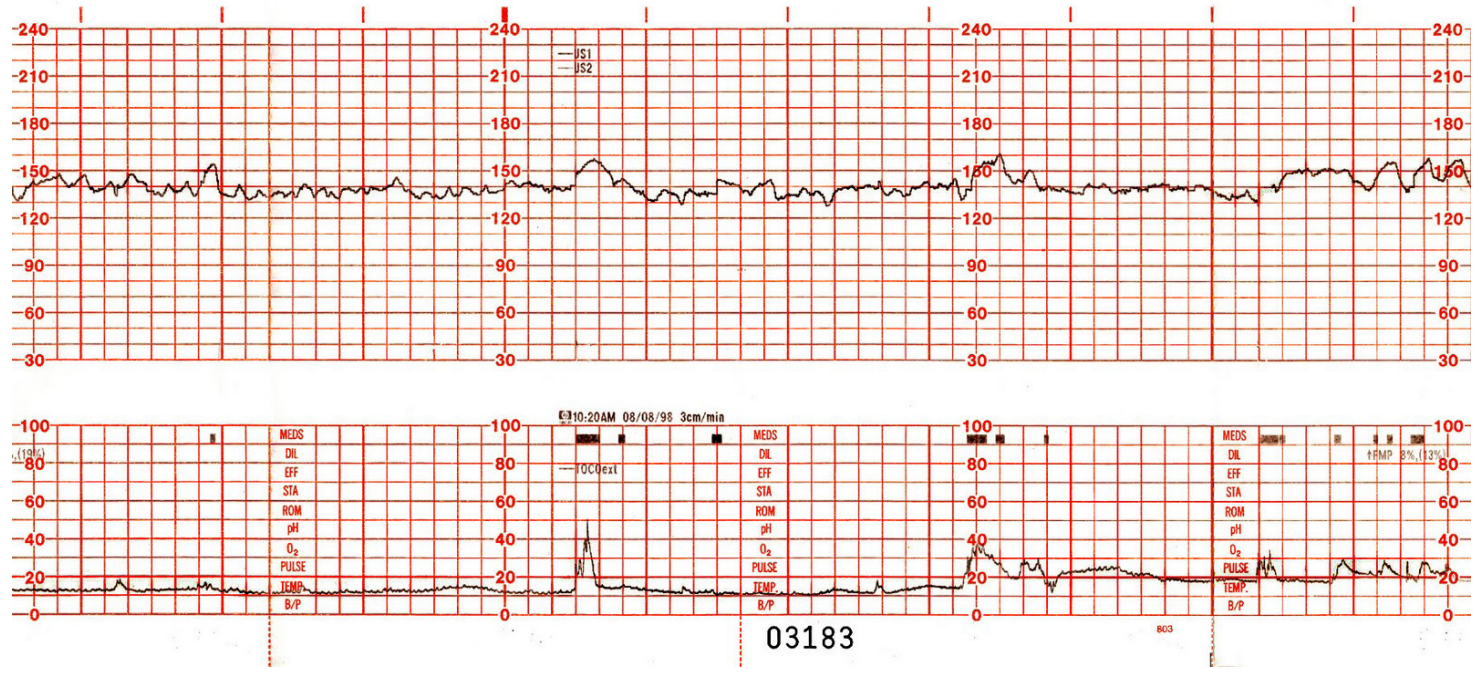

Figura $\mathbf{N}^{\circ 3}$. Variabilidad moderada (normal) de la frecuencia cardíaca fetal.

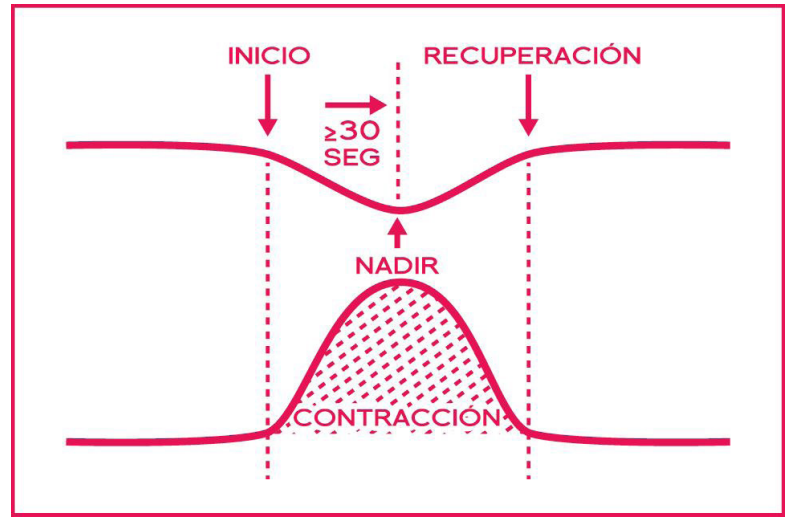

Figura $\mathbf{N}^{\circ}$ 4. Desaceleración temprana.

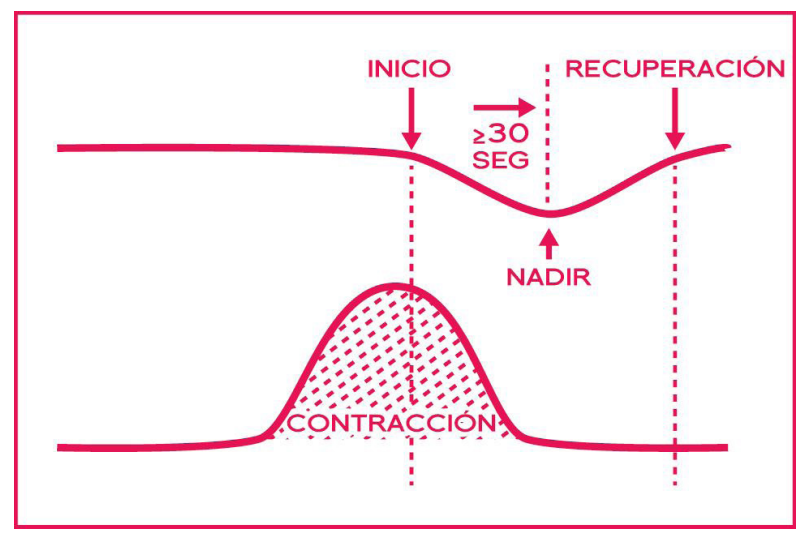

Figura $\mathbf{N}^{\circ}$ 5. Desaceleración tardía.

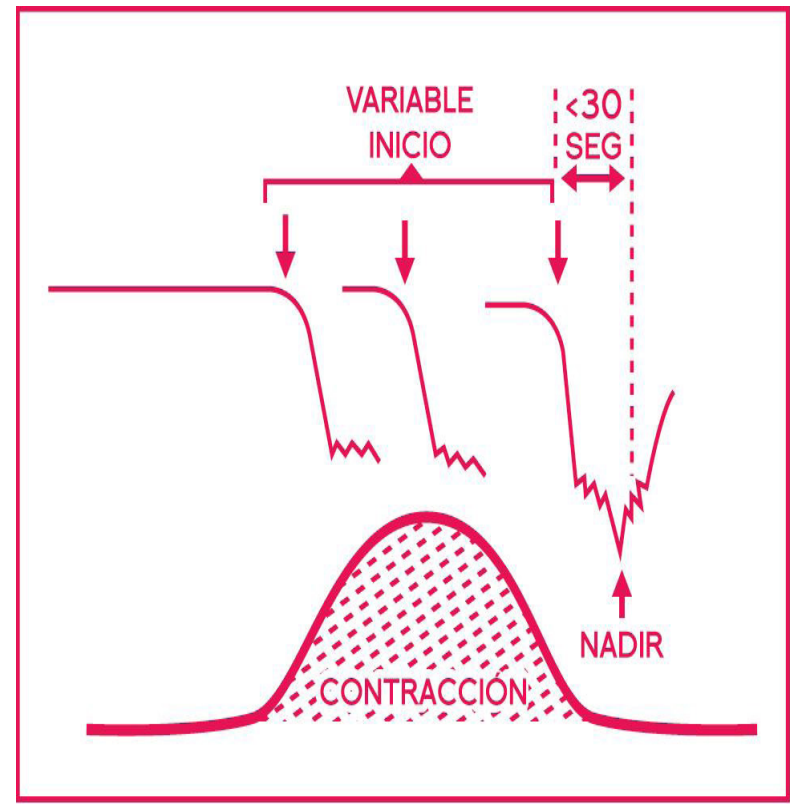

Figura $\mathbf{N}^{\circ} 6$. Desaceleraciones variables. 
La valoración del monitoreo fetal intraparto permite establecer un orden categórico, del cual se desprenden las conductas obstétricas a tener presentes (Tabla $N^{\circ} 3$ ). A su vez con toda esa información, se establece el denominado semáforo del monitoreo fetal, que debiese estar presente en todas las salas de trabajo de parto(Figura $\mathrm{N}^{0} 7$ ).

\begin{tabular}{|c|c|c|}
\hline \multicolumn{3}{|c|}{$\begin{array}{c}\text { TABLA N } 3 . \\
\text { MONITOREO INTRAPARTO CONTINUO. CATEGORIZACIÓN Y PLAN A SEGUIR }\end{array}$} \\
\hline CATEGORIZACIÓN & \begin{tabular}{|c|} 
CRITERIOS \\
\end{tabular} & $\begin{array}{l}\text { PLAN A SEGUIR } \\
\end{array}$ \\
\hline - Categoría I & $\begin{array}{l}\text { Monitoreo tranquilizador. Se continúa la } \\
\text { vigilancia del trabajo de parto. En el trazado } \\
\text { la FCF es normal, la variabilidad moderada y } \\
\text { no hay desaceleraciones tardías ni variables. } \\
\text { Las aceleraciones pueden estar presentes o } \\
\text { ausentes. }\end{array}$ & $\begin{array}{l}\text { Se registra cada hora en el } \\
\text { partograma los datos de la } \\
\text { actividad cardíaca fetal }\end{array}$ \\
\hline - Categoría II & $\begin{array}{l}\text { Indica la necesidad de vigilancia más } \\
\text { estrecha. El feto está en riesgo de hipoxia. Se } \\
\text { pueden encontrar ya sea alteración de la FCF, } \\
\text { aceleraciones o desaceleraciones. En algunos } \\
\text { casos será necesario iniciar reanimación fetal } \\
\text { intrauterina. }\end{array}$ & $\begin{array}{l}\text { 1. Coloque la paciente en } \\
\text { decúbito lateral izquierdo. } \\
\text { 2. Administre bolos de } \\
\text { cristaloides iso-osmolar } 250 \text { a } \\
500 \text { cc si la presión sistólica es } \\
\text { igual o menor de } 90 . \\
\text { 3. Administre acetaminofén } 1 \\
\text { gramo si hay fiebre mayor a } \\
38^{\circ} \mathrm{C} . \\
4 \text {. Suspenda la oxitocina y } \\
\text { evalúe el tono si hay hipertonía } \\
\text { o taquisistolia. Considere iniciar } \\
\text { tocolítico (terbutalina ). } \\
5 \text {. Realice tacto vaginal para } \\
\text { evaluar la dilatación y descartar } \\
\text { presencia de cordón. } \\
6 . \text { Realice amniotomia si las } \\
\text { membranas están íntegras, ello } \\
\text { permite evaluar las características } \\
\text { del líquido amniótico. }\end{array}$ \\
\hline - Categoría III & $\begin{array}{l}\text { Indica que se ha perdido el bienestar fetal y } \\
\text { hay alteración del estado acido-básico fetal. } \\
\text { Se debe intervenir inmediatamente y evaluar } \\
\text { la vía del parto para finalizar el embarazo } \\
\text { de acuerdo a la condición obstétrica. En el } \\
\text { registro se observará bradicardia, variabilidad } \\
\text { disminuida o ausente, desaceleraciones tardías } \\
\text { o variables recurrentes o patrón sinusoidal. }\end{array}$ & $\begin{array}{l}\text { 1. Coloque a la paciente en } \\
\text { decúbito lateral. } \\
\text { 2. Evalúe signos vitales } \\
\text { maternos: tensión arterial, pulso, } \\
\text { y temperatura } \\
\text { 3. Administre oxígeno en } \\
\text { mascarilla con bolsa reservoria a } \\
\text { 10 litros por minuto } \\
\text { 4.Administre cristaloides } 500 \text { cc } \\
\text { endovenosos a chorro } \\
\text { 5. Si hay hipertonía uterina, } \\
\text { contracción de dos o más } \\
\text { minutos, suspenda oxitocina e } \\
\text { inicie tocolíticos parenterales. } \\
\text { 6. Realice el tacto vaginal y } \\
\text { evalúe dilatación y descarte } \\
\text { prolapso del cordón umbilical. } \\
\text { 7. Realice masaje suave de la } \\
\text { calota fetal, sin la contracción. } \\
\text { 8. Informe al servicio de } \\
\text { Neonatología y Anestesiología. } \\
\text { 9. Seleccione la vía del parto. }\end{array}$ \\
\hline
\end{tabular}




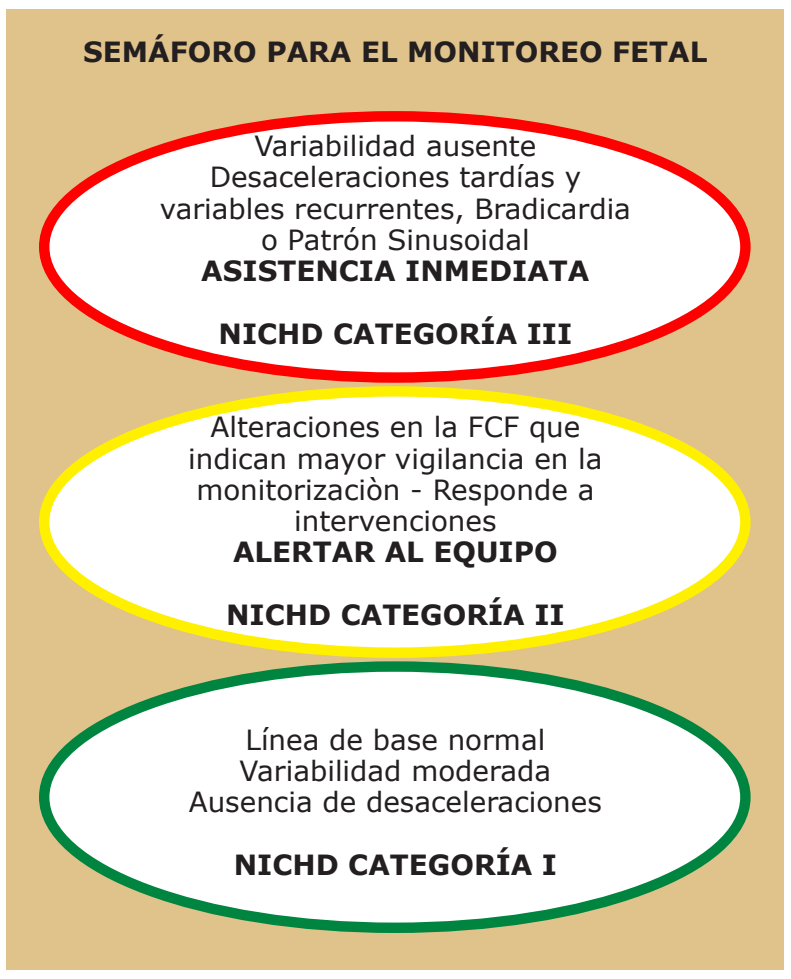

Figura $N^{\circ} 7$

\section{CASO CLÍNICO}

Paciente femenina de 29 años de edad. G3. P1. A1. C0. FUM del 26 de noviembre del 2014. Asiste al servicio de urgencias el 21 de agosto de 2015 con cuadro clínico de 5 horas de evolución consistente en dolor tipo cólico en hipogastrio irradiado a región lumbar. Niega otra sintomatología. Embarazo actual con tres consultas prenatales en puesto de salud, una por médico general y dos por enfermería. Trae ecografía única del 29 de junio que muestra embarazo de 31 semanas por biometría fetal. Hemograma del 29 de Diciembre señala hemoglobina de $10.7 \mathrm{~g} / \mathrm{dl}$ y hematocrito de $31 \%$. Al examen físico se encuentra paciente hemodinámicamente estable, sin alteraciones auscultatorias a nivel cardiopulmonar y abdomen globoso por útero grávido. AU: $34 \mathrm{~cm}$, producto longitudinal, cefálico, dorso derecho. FCF: 130 latidos por minuto y movimientos fetales presentes. Contractilidad uterina: $3 \times 40 \times 10$ de buena intensidad. A la especuloscopia, prueba de Tarnier negativa y no hay salida de sangre. Al tacto se encuentra cuello centrado, blando, Dilatación 5 centímetros y borramiento de $80 \%$. Membranas íntegras. Estación -1.
Se envía a sala de trabajo de parto y se realiza monitoreo electrónico. (Figura $\mathrm{N}^{\circ} 8$ )

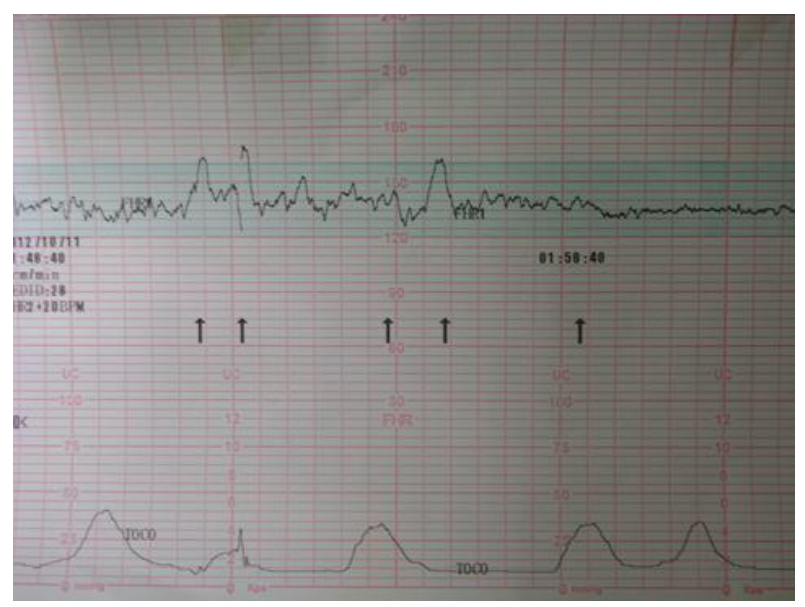

Figura $\mathbf{N}^{\circ} \mathbf{8}$

\section{Preguntas}

1. ¿Cuál es la frecuencia cardíaca fetal basal?

a) $120 \times \mathrm{min}$

b) $135 \times \mathrm{min}$

c) $140 x \min$

2. ¿Cómo considera la variabilidad de este trazado?
a) Ausente
b) Disminuida
c) Moderada

3. ¿Presenta desaceleraciones tardías o variables?
a) $\mathrm{Si}$
b)No

4. ¿Cuál es la categoría de este trazado?

a) Categoría I

b) Categoría II

c) Categoría III

\section{Respuestas}

Primera. (B). La frecuencia basal se determina cuando la misma permanece estable en un trazado al menos por 10 minutos. El papel de este monitoreo es de 5lat/min y en el centro se puede observar los números que establecen los latidos. En este trazado persiste el valor de 135 lat/min. Segunda. (C). La variabilidad es la variación natural que presenta la fetocardia, regida por la madurez del sistema nervioso autónomo (simpático y parasimpático) y es uno de los parámetros 
más importante del bienestar fetal en un trazado de monitoreo. Lo normal es que oscile de 6 a 25/lat .En este trazado la variabilidad es moderada o normal ya que sus oscilaciones están en los parámetros descritos. Tercera. (B). No se observan desaceleraciones. Las cuales se definen como caídas de la frecuencia cardiaca fetal, desde su línea basal iguales o mayores de 15 lat/min. Cuarta (A) El trazado es Categoría I ya que la FCF de base es normal, con variabilidad moderada y ausencia de desaceleraciones tardías o variables.

\section{LECTURAS RECOMENDADAS}

1. Alfirevic Z, Devane D, Gyte GML. Continuous car- diotocography (CTG) as a form of electronic fetal monitor- ing (EFM) for fetal assessment during labour. Cochrane Database Syst Rev. 2006;(3):CD006066

2. Cahill AG, Roehl KA, Odibo AO, Macones GA. Association of atypical decelerations with acidemia. Obstet Gynecol. 2012,120:1387-93.

3. American Academy of Pediatrics, American College of Obstetricians and Gynecologists. The Apgar Score. Policy statement. Pediatrics 2006;117(4):1444-47.

4. Barkovich AJ, Westmark K, Partridge C, Sola A, Ferriero DM. Perinatal asphyxia: MR findings in the first 10 days. AJNR Am J Neuroradiol 1995;16:427-38.

5. Blackwell SC, Grobman WA, Antoniewicz L, Hutchinson M, Gyamfi C. Interobserver and intraobserver reliability of the NICHD 3-Tier Fetal Hearth Rate Interpretation System. Am J Obstet Gynecol. 2011;205(4):378, e1-5.

6. Chau, V, Poskitt, KJ, Miller, SP. Advanced neuroimaging techniques for the term newborn with encephalopathy. Pediatr Neurol. 2009; 40:181.

7. Electronic fetal heart monitoring. Research guidelines for interpretation. National Institute of Child Health and Human Development Research Planning Workshop. Am J Obstet Gynecol. 1997; 177: 1385-90.

8. Freeman RK. Problems with intrapartum fetal heart rate monitoring interpretation and patient management. Obstet Gynecol. 2002;100:813-26.

9. Intrapartum fetal heart rate monitoring: Nomenclature, interpretation, and general management principles. ACOG Practice Bulletin. Number 106. Obstet Gynecol. 2009;114(1):192-202.

10. Korst LM, Phelan JP, Wang YM, Martin GI, Ahn MO. Acute fetal asphyxia and permanent brain injury: a retrospective analysis of current indicators. J Matern Fetal Med. 1999;8:101-6.

11. Low JA, Pickersgill H, Killen H, Derrick EJ. The prediction and prevention of intrapartum fetal asphyxia in term pregnancies. Am J Obstet Gynecol.2001;184:724-30.

12. Leijser LM, Vein AA, Liauw L, Strauss T, Veen S, Wezel-Meijler G. Prediction of shortterm neurological outcome in full-term neonates with hypoxic-ischaemic encephalopathy based on combined use of electroencephalogram and neuro-imaging. Neuropediatrics. 2007;38:219-27.

13. Liston R, Sawchuck D, Young D. Fetal health surveil- lance: antepartum and intrapartum consensus guideline. Society of Obstetrics and Gynaecologists of Canada; British Columbia Perinatal Health Program [published erratum appears in J Obstet Gynaecol Can 2007;29:909]. J Obstet Gynaecol Can. 2007;29(suppl 4):S3-56.

14. MacLennan A. A template for defining a causal relation between acute intrapartum events and cerebral palsy: international consensus statement. BMJ. 1999;319:1054-9.

15. Macones GA, Hankins GD, Spong CY, Hauth J, Moore T. The 2008 National Institute of Child Health and Human Development workshop report on electronic fetal moni- toring: update on definitions, interpretation, and research guidelines. Obstet Gynecol 2008;112:661-6.

16. Ment, LR, Bada, HS, Barnes $P$, et al. Practice parameter: neuroimaging of the neonate: report of the Quality Standards Subcommittee of the American Academy of Neurology and the Practice Committee of the Child Neurology Society. Neurology. 2002; 58:1726.

17. Miller David A., Miller Lisa A. Electronic fetal heart rate monitoring: applying principles of patient safety. Am J Obstet Gynecol. 2012: 278-83

18. Moster D, Lie RT, Irgens LM, Bjerkedal T, Markestad T. The association of apgar score with subsequent death and cerebral palsy: a population-based study in term infants. J Pediatr 2001;138:798-803.

19. Parer JT, Ikeda T. A framework for standardized manage- ment of intrapartum fetal heart rate patterns. Am J Obstet Gynecol 2007;197(26):1-26.

20. Pasternak JF, Gorey MT. The syndrome of acute near-total intrauterine asphyxia in the term infant. Pediatr Neurol 1998;18:391-8.

21. Roger K. Freeman, Thomas J. Garite, Michael P Nageotte. Fetal Heart Rate Monitoring. 3a Edición. Philadelphia: Lippincott Williams \& Wilkins, 2003.

22. Royal College of Obstetricians and Gynaecologists. The use of electronic fetal monitoring: the use and interpretation of cardiotocography in intrapartum fetal surveillance. Evidence-based Clinical Guideline No. 8. London (UK): RCOG;2001.http://www.rcog.org.uk/files/rcog-corp/uploaded-files/NEBEFMGuidelineFinal2may2001.pdf (Level III)

23. Ruis KA, Ruis KA, Lehmann CU, Northington FJ, Lin DD, Graham EM. Neonatal brain imaging and 
the identification of metabolic acidemia and hypoxic-ischemic encephalopathy. J Matern Fetal Neonatal Med 2009;22(10):823-8.

24. Williams Keith $P$, Galernau France. Intrapartum fetal heart rate patterns in the prediction of neonatal acidemia. Am J Obstet Gynecol. 2003; 188(3): 820-23.

25. Yudkin PL, Johnson A, Clover LM, Murphy KW. Assessing the contribution of birth asphyxia to cerebral palsy in term singletons. Paediatr Perinat Epidemiol.1995;9(2):156-70.

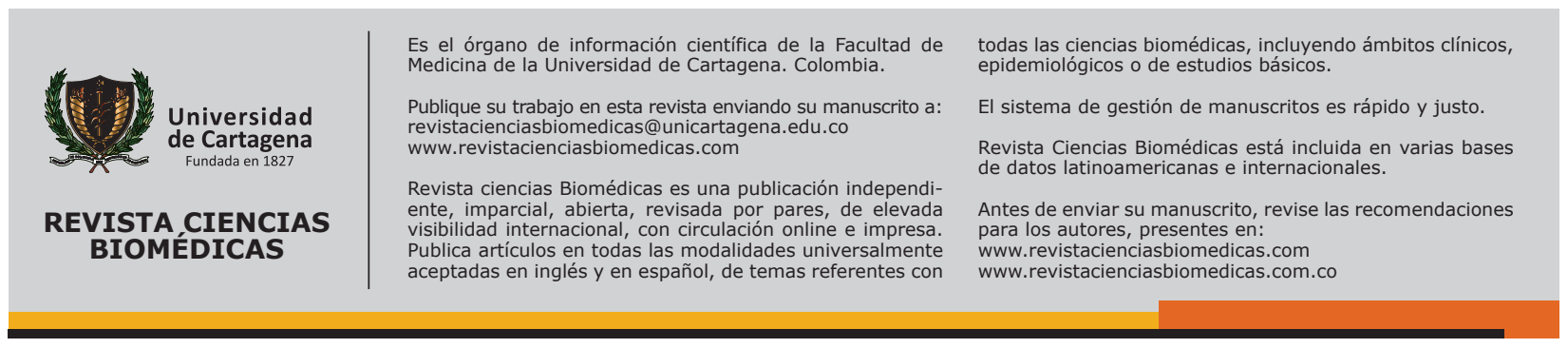

